worker's maximum BL, were found for brain cancer (malignant and benign combined), Hodgkins's lymphoma, lung cancer, and rectal cancer, while significant negative trends were found for colon cancer and melanoma. A borderline significant positive trend $(0.05 \leq \mathrm{p} \leq 0.10)$ was found for esophageal cancer. Significant interactions by country were found only for lung cancer, with Finland showing a strong positive trend and the UK showing only a modest trend. However, in general trends were marked in Finland and weak or inconsistent in the UK.

Conclusions We found strong positive incidence trends with increasing blood lead level, for several outcomes in internal analysis. Two of these, lung and brain cancer, were a priori suspected sites. Two of these outcomes are associated with smoking (lung and esophageal cancer), for which we had no data; however, we had no a priori reason to believe smoking differed between workers with different BL levels.

\section{C.5 ASSESSMENT AND ASSIGNMENT OF EXPOSURE TO ASBESTOS FOR AN INDUSTRIAL COHORT OF CHRYSOTILE MINERS AND PROCESSORS}

\begin{abstract}
${ }^{1}$ Hans Kromhout ${ }^{*},{ }^{2}$ Eleonora Feletto, ${ }^{3}$ Monika Moissonnier, ${ }^{4}$ Sara J Schonfeld, ${ }^{3}$ Ann Olsson, ${ }^{5,6}{ }^{2}$ vvgeny $\vee$ Kovalevskiy, ${ }^{5,6}$ Igor V Bukhtiyarov, ${ }^{7}$ Sergey $V$ Kashanskiy, ${ }^{3}$ Joachim Schüz. ${ }^{1}$ Utrecht University, Utrecht, Netherlands; ${ }^{2}$ Cancer Research Division, Cancer Council New South Wales, Woolloomooloo, Australia; ${ }^{3}$ Section of Environment and Radiation, International Agency for Research on Cancer, Lyon, France; ${ }^{4}$ Division of Cancer Epidemiology and Genetics, National Cancer Institute, National Institutes of Health, Bethesda, USA; ${ }^{5}$ Izmerov Research Institute of Occupational Health, Moscow, Russian Federation; ${ }^{6}$ 5I.M. Sechenov First Moscow State Medical University (Sechenov University), Russian Federation; ${ }^{7}$ Yekaterinburg Medical Research Center for Prophylaxis and Health Protection in Industrial Workers, Yekaterinburg, Russian Federation
\end{abstract}

\subsection{6/OEM-2019-EPI.21}

Introduction Historical dust concentrations are available for an occupational cohort study of workers active for 12 months or more between 1975 and 2010 in a chrysotile mine and processing factories in Asbest, Russian Federation. Their occupational histories were ascertained back to as early as the 1930s. A cohort specific job-exposure matrix (JEM) to estimate exposure to asbestos dust and fibre was elaborated.

Methods Almost 100000 recorded dust concentrations were used to develop an asbestos dust JEM and previously derived conversion factors were applied to estimate an asbestos fibre JEM. Where dust concentrations were not available, linear mixed models were used to impute missing data. Both JEMs were applied to the occupational histories of over 30000 individual workers (over 35\% female workers) based on job title and year worked.

Results Assigned exposures varied over time with higher levels in the earlier years of activity. Approximately $97 \%$ of 200000 person-years in the factories and $89 \%$ of 315000 person-years in the mine had exposure assigned based on actual measurements. The median cumulative dust exposure for the exposed cohort was almost $50 \mathrm{mg} / \mathrm{m} 3$-years, with women slightly lower than men. The median cumulative fibre exposure for was 37 fibres/cm3-years for both men and women.
Discussion and conclusion A key strength of this study is the availability of high-quality measurement data covering workers' occupational histories. The dust and fibre JEMs enable estimation of annual profession-specific exposure levels that will form the basis of quantitative exposure estimates in the study and consequently quantitative exposure-response analyses.

\section{C.6 IS ADJUSTMENT FOR SMOKING NEEDED IN A COHORT STUDY OF CANCER MORTALITY AMONG CHRYSOTILE ASBESTOS FACTORY AND MINE WORKERS?}

${ }^{1}$ Ann Olsson*, ${ }^{1}$ Madar Talibov, ${ }^{2}$ Evgeny V Kovalevskiy, ${ }^{1}$ Graham Byrnes, ${ }^{3}$ Sara Schonfeld, ${ }^{4}$ Eleonora Feletto, ${ }^{5}$ Sergey V Kashanskiy, ${ }^{1}$ Monika Moissonnier, ${ }^{1}$ Evgenia Ostroumova,

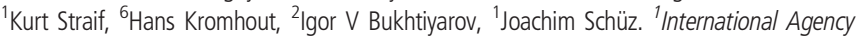
For Research On Cancer, Lyon, France; ${ }^{2}$ Izmerov Research Institute of Occupational Health, Moscow, The Russian Federation; ${ }^{3}$ National Cancer Institute, Division of Cancer Epidemiology and Genetics, Radiation Epidemiology Branch, Bethesda, USA; ${ }^{4}$ Cancer Council NSW, Woolloomooloo, Australia; ${ }^{5}$ Yekaterinburg Medical Research Center for Prophylaxis and Health Protection in Industrial Workers, Yekaterinburg, The Russian Federation; ${ }^{6}$ Institute for Risk Assessment Sciences, Utrecht, The Netherlands

\subsection{6/OEM-2019-EPI.22}

Introduction A retrospective cohort study of 35840 employees is currently being conducted in a chrysotile mine and its processing facilities in Asbest, the Russian Federation. The primary aim is to quantify exposureresponse relationships for cancers potentially associated with chrysotile exposure. Some of those cancers are also tobacco-related; however individual-level information on tobacco use is not available for the full cohort. To address this gap, a cross-sectional study of current and retired workers from JSC Uralasbest was conducted to assess the relationship between smoking status and workers' exposure to chrysotile.

Methods Self-administered questionnaires were completed by current workers during meetings organized by occupational safety specialists. Retired workers filled in questionnaires during Veterans' meetings or were interviewed via telephone or at home. Estimates of exposure to chrysotile were available for 999 current and 3795 retired workers who were linked to the cohort study.

Results Among the 7451 respondents $(n=3698$ men and $n=3753$ women), $66 \%$ of men and $9 \%$ of women were ever-smokers. Smoking prevalence was stable across birth decades in men, but increased from $<10 \%$ in women born before 1960 to $30 \%$ in women born after 1980. Among ever-smokers, men smoked more cigarettes per day than women. The smoking prevalence was similar in exposed and non-exposed men, and did not increase with exposure category. The greatest difference in the proportion of smokers among women was observed between non-exposed (4\%) and all exposed categories combined $(7.5 \%)$, whereas there was little variation across categories of exposure (7\%-8\%). Overall, the self-reported smoking prevalence may have been underestimated, especially in the older age categories due to survivor bias.

Conclusion While no adjustments for smoking among men appear necessary in the future analyses, including smoking propensity by birth cohort for women may be useful. 